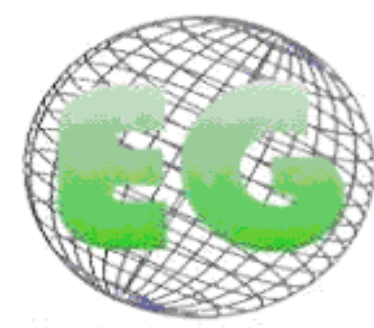

ISSN 1695.6141 $N^{\circ} 26$

\title{
Nivel de conocimientos y práctica adquiridos durante un curso-taller de terapia intravenosa
}

Level of knowledge and practice acquired during a workshop on intravenous therapy

\section{*Resendiz Gutiérrez, MG., **Muñoz Torres, T de J.}

\begin{abstract}
*Maestra en Administración de la Atención de Enfermería. Jefa de Enfermeras del Hospital General ISSSTE Rioverde, San Luis Potosí. E-mail: resendizgutierrez@yahoo.com.mx "**Estudiante de la Licenciatura en Enfermería de la Unidad Académica Multidisciplinaria Zona Media de la Universidad Autónoma de San Luis Potosí. México.
\end{abstract}

Palabras clave: terapia intravenosa; conocimiento; práctica Keywords: intravenous therapy; knowledge; practice.

\section{RESUMEN}

Objetivo: Analizar el nivel de conocimientos adquiridos durante el desarrollo de un curso-taller de terapia intravenosa y su relación con la aplicación práctica del procedimiento.

Material y métodos: Estudio cuasiexperimental, descriptivo y de corte transversal. Muestra total de 30 enfermeras operativas de los servicios donde se realiza esta técnica. Se utilizó como instrumentó de evaluación un cuestionario y una guía de observación estructurada que se aplicó en 3 ocasiones. El procesamiento estadístico se realizo a través del programa Excel y SPSS, utilizándose un análisis descriptivo.

Resultados: el $63.3 \%$ del profesional de enfermería obtuvo un nivel medio de conocimiento, en lo que respecta al mantenimiento y prevención de complicaciones predominó el nivel alto con un $83.8 \%$.

Conclusión: El presente estudio permite identificar que la preparación constante del personal de enfermería mediante la aplicación de cursos-talleres incrementa de manera positiva el manejo de conocimiento y la práctica pero sobre todo garantiza al usuario una atención con el menor riesgo y una mayor calidad.

\section{ABSTRACT}

Aim: To analyse the level of knowledge acquired during the development of a workshop on intravenous therapy and its relation to the practical application of the procedure.

Material and methods: quasi-experimental, descriptive, transversal study applied to 30 operational nurses who worked in the services where this technique is carried ou. For evaluation a questionnaire and a structured guide of observation was used, which was applied on 3 occasions. The statistical analysis was performed in Excel and SPSS, using descriptive analysis. 
Results: $63.3 \%$ of nursing professional obtained a mean level of knowledge, with regard to maintenance and prevention of complications, thee was a high level, $83.8 \%$.

Conclusion: This study identifies that the constant preparation of nurses through the implementation of workshops increases positively management knowledge and practice but especially guarantees the user a lower risk and higher quality attention.

\section{INTRODUCCIÓN}

El uso de catéteres intravasculares es indispensable en la práctica diaria de los profesionales de enfermería, pues su aplicación se ha generalizado, su inserción anatómica es muy variada y su duración de amplísimos rangos, en respuesta a las tareas asistenciales y patológicas. Se tiene de conocimiento a través de diversos estudios que la prevalencia de vías vasculares insertadas en los pacientes ingresados al área hospitalaria es muy diversa debido a que un $45 \%$ de estas se realizan a nivel periférico, $5.5 \%$ a nivel central y solo un $2.5 \%$ son catéteres de nutrición parenteral total ${ }^{1}$.

Con frecuencia el manejo de los pacientes hospitalizados requiere de la instauración de una vía intravenosa para la reanimación aguda, hidratación, control de dolor, observación del paciente, transfusión sanguínea y recolección de muestras sanguíneas; en respuesta a ello, en la actualidad se han desarrollado diversas técnicas para el logro de estos objetivos, sin embargo, la inserción de un catéter venoso periférico es la primera elección, debido a que es un procedimiento sencillo, rápido, eficaz y con el mínimo de complicaciones para el paciente, siempre y cuando sea realizado por profesionales de enfermería calificados y con destreza ${ }^{2}$.

La terapia intravenosa es un procedimiento que consiste en la inserción de un catéter en la luz de la vena, a través del cual se infunden líquidos, medicamentos, sangre o sus derivados. Es considerada como la vía más rápida y efectiva para la administración de lo antes mencionado. Dicho procedimiento consta de 5 pasos esenciales: valoración del sitio, selección del catéter, preparación del sitio de punción, inserción, mantenimiento y prevención de complicaciones ${ }^{3}$.

La Organización Mundial de la Salud en 2003 elaboró una guía práctica para la prevención de infecciones intrahospitalarias en donde refleja la importancia del lavado de manos, uso de guantes, la limpieza del entorno hospitalario y la calidad de los procedimientos ${ }^{18}$; pues la terapia intravenosa es uno de los más comunes y que puede desencadenar diversos problemas durante la inserción y mantenimientos de una vía permeable, pues en México, según datos proporcionados por la Red Hospitalaria para la vigilancia epidemiológica, las infecciones nosocomiales ocupaban el primer lugar de las principales causas de muerte y de este total de infecciones bacterianas, el $75 \%$ se asociaba a la utilización de catéteres y soluciones intravenosas, lo que conlleva a una serie de alteración, no solo a nivel institucional sino también en el aumento de la estancia hospitalaria y del riesgo terapéutico ${ }^{5}$.

Diversas investigaciones realizadas a lo largo de varios años, indican que la buena práctica de este procedimiento desencadena en la prevención de complicación, por ello la Comisión Nacional de Arbitraje Médico y el grupo de validación externa coordinado por la Comisión Interinstitucional de Enfermería, emitió unas recomendaciones sobre la aplicación de la terapia intravenosa, lo que origino que este se convirtiera en un indicador de calidad de la atención enfermera, por su importancia en el control de riesgos y daños al paciente ${ }^{5}$.

Por lo que en el Programa Nacional de Salud 2000-2006 estableció estrategias esenciales para la Calidad de los Servicios de Salud, en donde unos de los puntos importantes era la adecuada realización de los procedimientos, lo que originó que la Secretaría de Salud 
elaborara un formato con el cual se evalúa la práctica enfermera durante el desarrollo del procedimiento con el propósito de evitar las complicación potenciales derivadas de una inserción venosa carente de principios prácticos ${ }^{6}$. Por su parte el Programa Nacional de Salud 2007-2012, para prestar servicios de salud con calidad y seguridad se planteó como metas acreditar al 100\% de las unidades de salud e implementar medidas de prevención de eventos adversos, para garantizar la seguridad de los pacientes en un $60 \%{ }^{7}$.

En la actualidad se reconoce la necesidad de establecer mecanismos permanentes de vigilancia epidemiológica que permitan el manejo ágil y eficiente de la información necesaria para la prevención y control de la infección intrahospitalarias, por lo que se considera indispensable homogenizar los procedimientos y criterios institucionales que orienten $y$ faciliten el trabajo del profesional. Teniendo en cuenta estos razonamientos y las situaciones antes mencionadas, el presente estudio se planteó como objetivo analizar el nivel de conocimientos adquiridos durante el curso-taller de terapia intravenosa y su relación con el uso en la práctica, por las enfermeras del Hospital General del Instituto de Seguridad y Servicios Sociales para los Trabajadores del Estado (ISSSTE).

\section{MATERIAL Y MÉTODOS}

\section{Tipo de estudio}

Este fue un estudio descriptivo, de corte transversal y cuasiexperimental debido a que no se utilizó un grupo control ${ }^{8}$, se llevo a cabo del 1 de Septiembre al 8 de Diciembre de 2005 en el Hospital General del Instituto de Seguridad y Servicios Sociales para los Trabajadores del Estado en la ciudad de San Luis Potosí (México) con profesionales de enfermería.

\section{Muestra}

Como técnica de recolección de datos, se tomó una muestra total de 30 enfermeras seleccionadas por muestro aleatorio simple y de conveniencia.

\section{Instrumentos}

Para obtener la información necesaria, se utilizaron dos instrumentos, un cuestionario tipo examen para medir el nivel de conocimientos de las enfermeras en relación con la terapia intravenosa; y una guía de observación con la cual se medía el nivel de aplicación de dichos conocimientos en el momento de la instalación de la venoclisis, mismo que contemplaba un apartado para medir el nivel de conocimientos y aptitudes relacionadas con el mantenimiento y prevención de complicaciones.

El cuestionario constó de 28 ítems, los cuales fueron divididos en 5 indicadores (precauciones universales, técnicas de inserción del catéter, preparación del sitio, mantenimiento de la venoclisis y prevención de complicaciones). En lo que respecta a la guía de observación, esta contemplaba 2 apartados, una que correspondía a las precauciones universales-instalación de venoclisis, y la segunda su mantenimiento y prevención de complicaciones. Ambos instrumentos fueron aplicados pre y post curso-taller.

\section{Análisis de datos}

Para el análisis estadístico se empleó el programa Excel y SPSS versión 18 con el cual se analizaron frecuencias absolutas y relativas por indicador, a través de tablas de frecuencias, porcentajes, desviación estándar, media y porcentajes. 


\section{RESULTADOS}

La tabla 1 muestra los resultados obtenidos de la muestra objeto de estudio de nivel operativo en relación con los aspectos sociodemográficos.

Tabla 1. Datos sociodemográficos de la muestra objeto de estudio

\begin{tabular}{|c|c|c|c|}
\hline \multicolumn{2}{|c|}{ Característica sociodemográficas } & \multicolumn{2}{|c|}{ Nivel de conocimientos } \\
\hline $\begin{array}{l}\text { Genero } \\
\text { Femenino }\end{array}$ & $100 \%$ & \multicolumn{2}{|c|}{$\begin{array}{l}\text { Evaluación inicial } \\
\text { El conocimiento general antes del curso fue de 5.5. }\end{array}$} \\
\hline \multicolumn{2}{|c|}{$\begin{array}{l}\text { Edad } \\
\text { Mínimo de } 24 \text { años, máximo de } 58 \\
\text { años, con un promedio de } 43.5 \text { y una } \\
\text { DE de }+/-8.25 \text { años. }\end{array}$} & \multicolumn{2}{|c|}{$\begin{array}{l}\text { Distribución inicial de conocimientos } \\
\text { El } 50 \% \text { tuvo una calificación menor de } 5.7 \% \text {, con } \\
\text { una DE de +/- } 1.16 \text {, mínima de } 3 \text { y máxima de } 8 \text {. } \\
\text { Un } 25 \% \text { obtuvo una calificación mayor de } 6.4 \text {. }\end{array}$} \\
\hline $\begin{array}{l}\text { Tipo de contrato } \\
\text { Base } \\
\text { Eventual }\end{array}$ & $\begin{array}{l}83.3 \% \\
16.7 \%\end{array}$ & \multicolumn{2}{|c|}{$\begin{array}{l}\text { Evaluación posterior } \\
\text { El promedio general se incremento a } 7.75\end{array}$} \\
\hline \multicolumn{2}{|c|}{$\begin{array}{l}\text { Servicios hospitalarios } \\
\text { Urgencias, Quimioterapia, Unidad de } \\
\text { Cuidado Intensivos, Quirófano, } \\
\text { Neonatología, Medicina Interna, } \\
\text { Gineco-obstetricia, } \\
\text { especializada, Hemodiálisis y Pediatría. }\end{array}$} & \multicolumn{2}{|c|}{$\begin{array}{l}\text { Distribución posterior de conocimientos } \\
\text { El } 50 \% \text { obtuvo una calificación superior al } 7.9 \text {, } \\
\text { con una desviación estándar de } 0.87 \text {, mínima de } 6 \\
\text { y máxima de } 9 \text {. } \\
25 \% \text { de la muestra objeto de estudio tiene una } \\
\text { calificación menor de } 7 \text {. }\end{array}$} \\
\hline $\begin{array}{l}\text { Grado académico } \\
\text { Auxiliar } \\
\text { General } \\
\text { Licenciada } \\
\text { Especialista }\end{array}$ & $\begin{array}{l}6.7 \% \\
73.3 . \% \\
10 \% \\
10 \% \\
\end{array}$ & $\begin{array}{l}\text { Precauciones universales } \\
\text { Alto } \\
\text { Medio } \\
\text { Bajo }\end{array}$ & $\begin{array}{l}16.7 \% \\
66.7 \% \\
16.7 \%\end{array}$ \\
\hline $\begin{array}{l}\text { Distribución por turno } \\
\text { Matutino } \\
\text { Vespertino } \\
\text { Jornada Acumulada* }\end{array}$ & $\begin{array}{c}56.7 \% \\
20 \% \\
23.3 \% \\
\end{array}$ & $\begin{array}{l}\text { Instalación venoclisis } \\
\text { Alto } \\
\text { Medio } \\
\text { Bajo }\end{array}$ & $\begin{array}{l}63.3 \% \\
30 \% \\
6.7 \% \\
\end{array}$ \\
\hline \multirow{2}{*}{\multicolumn{2}{|c|}{$\begin{array}{l}\text { Antigüedad laboral } \\
\text { Mínimo de } 1 \text { año, máximo de } 21 \text { años, } \\
\text { varianza de } 22.04 \text { y una DE de }+/-4.69 \\
\text { años. }\end{array}$}} & \multicolumn{2}{|c|}{$\begin{array}{l}\text { Mantenimiento y prevención de complicaciones } \\
\text { Predomino el nivel alto, debido a que en } \\
\text { mantenimiento de la instalación de la venoclisis se } \\
\text { obtuvo un } 83.8 \% \text { y en prevención de } \\
\text { complicaciones un } 88.3 \% \text {. }\end{array}$} \\
\hline & & $\begin{array}{l}\text { Conocimiento post curso-taller } \\
\text { Alto } \\
\text { Medio }\end{array}$ & $\begin{array}{l}63.3 \% \\
36.7 \%\end{array}$ \\
\hline
\end{tabular}

* Corresponde a un turno de 12 horas de trabajo por día (sábado y domingo)

En lo que respecta al conocimiento de las enfermeras sobre precauciones universales por indicador se puede observar en el gráfico 1 que predominó el nivel de conocimientos alto salvo en el indicador que hace mención al uso de guantes, pues un pequeño porcentaje se ubicó en un nivel bajo. En tanto a lo referente al conocimiento de la enfermera sobre la instalación de una venoclisis, en el gráfico 2 se observa que en mayor porcentaje se ubica en un nivel alto de conocimientos, sin embargo en el indicador relacionado con la preparación del sitio de punción una porción importante de enfermeras se ubicó en el nivel bajo de conocimientos. 
Grafico 1. Relacion del conocimiento pre y post curso de terapia intravenosa realizado en el hospital general del issste.

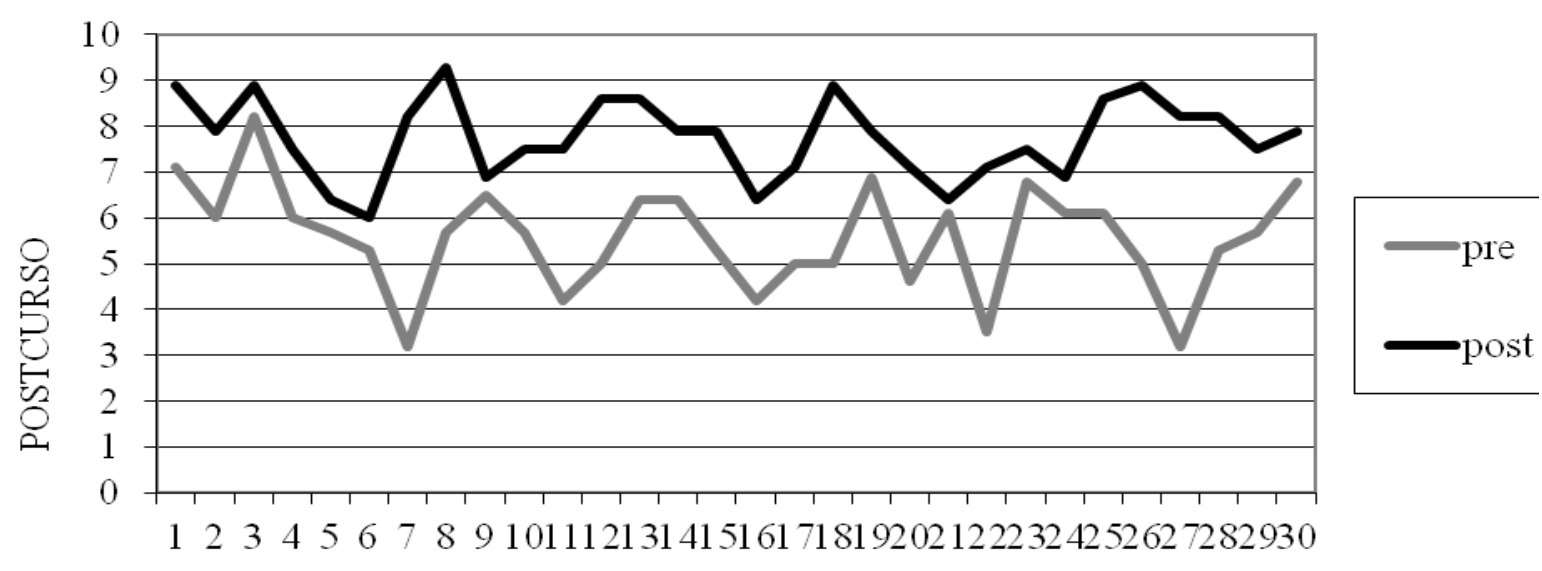

PRECIJRSO

Grafico 2. Nivel de conocimientos de las enfermeras por indicador en instalacion de terapia intravenosa. hospital general del ISSSTE.

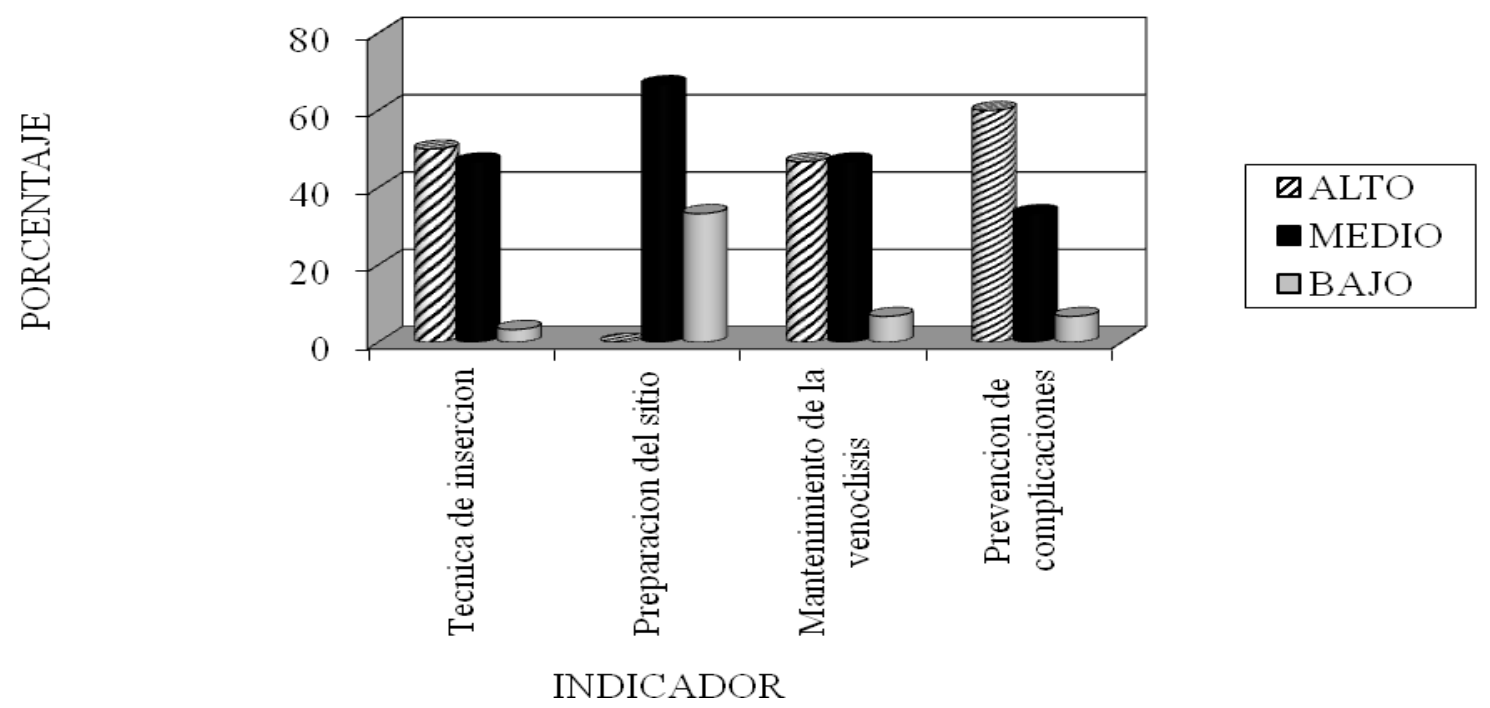

El uso de estas precauciones universales por observación y por indicador se muestra que el lavado de manos y uso de cubre bocas se ubicó en un nivel alto de conocimientos durante la primera observación y muy baja en el resto de las observaciones, sin embargo el uso de lentes siempre estuvo bajo durante las tres observaciones (gráfico 3). 
Grafico 3. Uso de precauciones universales en la instalacion de una venoclisis. hospital general del ISSSTE.

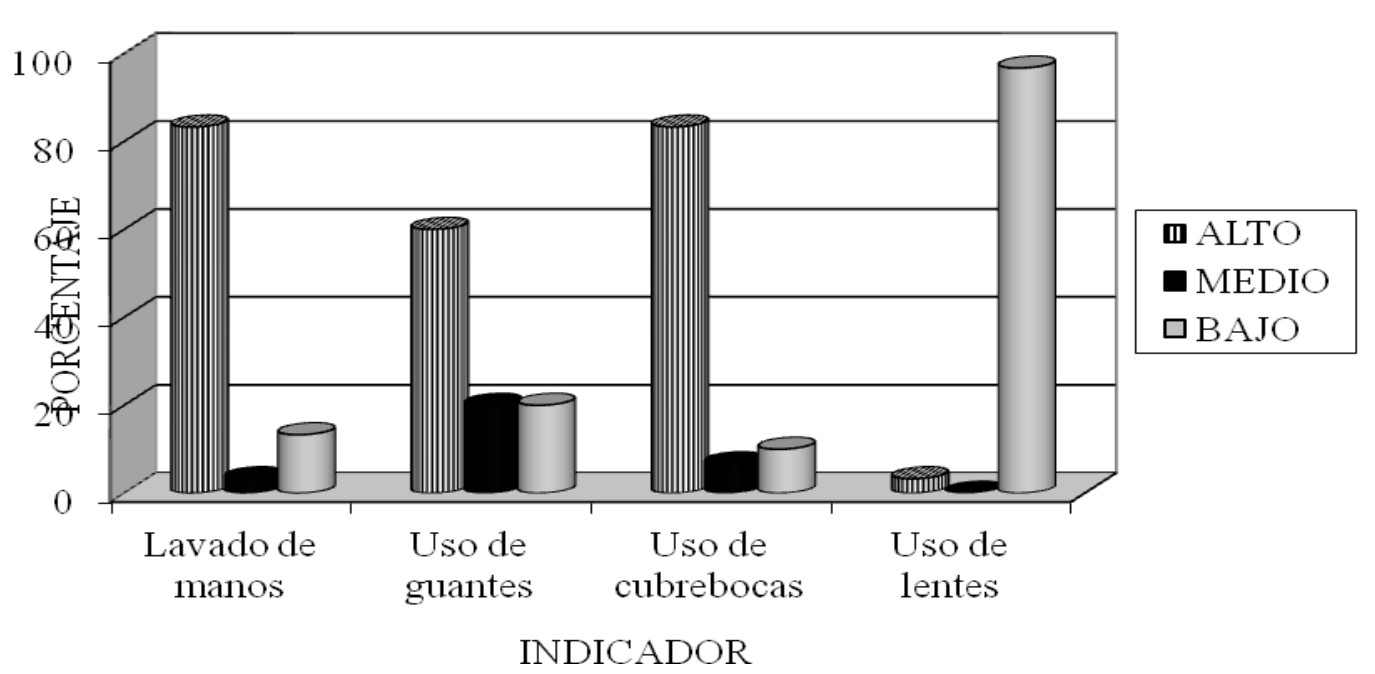

\section{DISCUSIÓN}

Los resultados relacionados con el promedio general antes y después de que el profesional de enfermería asistiera al curso-taller de terapia intravenosa reflejan lo indispensable que resulta la impartición de cursos que favorezcan la práctica clínica homogénea. Sin embargo, en un estudio previo referente al nivel de conocimientos y uso de las precauciones universales en el servicio de urgencias del hospital General del ISSSTE en San Luis Potosí, se concluye que no hay una asociación estadísticamente significativa entre conocimiento y uso ${ }^{9}$.

En lo que respecta al lavado de manos, Peña en su estudio realizado en el servicio de urgencias del Hospital General Regional 72 del Instituto Mexicano del Seguro Social, identificó que esta práctica es baja ${ }^{10}$, lo que resulta contrastante pues es considerada una medida muy económica, sencilla y eficaz para la prevención de infecciones nosocomiales, pues su importancia radica en que las manos son el instrumento más importante que se tiene, sin embargo, también puede servir como vehículo de transporte de gérmenes, desde diversos ángulos (enfermera-paciente, paciente-enfermera, paciente-paciente y enfermeraenfermera $)^{11}$. Además es sabido que en la manos existe flora residente y transitoria, tanto para bacterias gran positivas como gran negativas; por ello un simple pero eficaz lavado de manos siguiendo la técnica, que implica un tiempo mínimo de enjabonado de 15 a 30 segundos y dando una especial atención a uñas, dedos, espacios interdigitales, nudillos y palmas, se elimina la mayor parte de bacterias ${ }^{12}$. Aunado a lo anterior, resulta de suma importancia el uso de guante en el personal de salud al momento de realizar esta técnica, pues su uso tiene como objetivo evitar que las manos de las personas sean colonizadas de forma transitoria por bacterias que originen infecciones y que complican la evolución del paciente ${ }^{13}$; recordando que el uso de guantes en ningún momento sustituye el lavado de manos, pues este procedimiento se realiza antes y después de la técnica.

El uso de cubre bocas se ha considerado como un medio de protección para el personal de salud y pacientes al impedir el contacto entre las gotas de saliva o técnicas que puedan generar salpicaduras, de esta manera las mucosas conjuntivales nasales y orales del 
personal enfermero, se protegen de secreciones, sangre o fluidos corporales procedentes del paciente que pudieran estar infectados ${ }^{13}$.

Por otro lado el objetivo de la preparación del sitio es disminuir la flora bacteriana mediante el uso de técnicas de asepsia y soluciones antisépticas, ya que existen factores de riesgo para la contaminación e infecciones pues está relacionado entre otros aspectos con la susceptibilidad del huésped, la integridad cutánea, tiempo de permanencia del catéter, tiempo de hospitalización, el lugar de la inserción, etc ${ }^{14}$.

En un estudio realizado en el 2003 en los Servicios de Medicina y Cirugía Pediátrica en la Clínica Infantil Stauros Barcelona España en un periodo comprendido entre los meses de diciembre del 2000 a marzo del 2001 demostró que las vías venosas periféricas canalizadas en el dorso de la mano presentan menos complicaciones que las canalizadas en el plexo braquial $^{15}$, aspecto transcendental en el control de las complicaciones de la terapia intravenosa. Aunado a lo antes expuesto Pearson, Dougherty, Millam, Inwood, Taylor, Rees, Campbell, Kiernan analizaron el uso de antisepsia y asepsia previa a una punción, y coincidieron en que un adecuado lavado de manos, la utilización de guantes y el uso de yodopovidona al $10 \%$, alcohol al $70 \%$ o tintura de yodo al $2 \%$ son las mejores medidas para la preparación de la piel previa a una punción ${ }^{19}$, pues los agentes causales de infecciones en el torrente sanguíneo son el estafilococo coagulasa, estafilococos aureus, enterococo y cándida (flora residente); de ahí la importancia de medidas de prevención y apego estricto a técnicas de asepsia y antisepsia; ya que esta intervención rompe la primera barrera de protección $^{14}$.

En relación al conocimiento presente en el personal de enfermería, los resultados son muy similares al estudio de Carvajal en donde concluye que las enfermeras aplican las medidas de asepsia y antisepsia antes, durante y después de la intervención a pacientes con vía venosa central $^{16}$.

El mantenimiento del catéter tiene como objetivo evitar infecciones, flebitis, trombosis y bacteremia, por lo que al análisis de los resultados estos se apegan a lo estipulado en la Norma Oficial Mexicana 002 que establece que los equipo de infusión deben ser rotulados con la flecha, hora y nombre de la persona que lo instaló así como todas las medidas relacionadas con el tiempo de instalación, cambio de equipo y medidas de higiene al momento de aplicar medicamentos ${ }^{4}$.

A pesar de que se reconoce a la infección nosocomial como una complicación donde se conjugan diversos factores de riesgo y susceptibilidad, en la mayoría de los casos debe prevenirse, se debe señalar que existen casos en los que la infección nosocomial se presenta debido a condiciones inherentes al huésped ${ }^{3}$. De ahí la importancia de haber considerado la prevención de complicaciones en donde predominó el nivel alto en ambas variables por lo que se infiere que la ausencia de complicaciones se debe a que el personal aplica las medidas preventivas necesarias para mantener los catéteres periféricos cortos, estos resultados difieren de lo encontrado por Salazar en el hospital de Chihuahua México en pacientes de diferentes servicios en donde se observaron casos de morbilidad asociados a infecciones nosocomiales en donde uno de los factores considerados fue la venoclisis ${ }^{17}$.

\section{CONCLUSIONES}

La protección de la salud es un derecho social; en este sentido, la capacitación como un proceso continuo de enseñanza-aprendizaje, mediante el cual se desarrollan las habilidades y destrezas de los servidores, permite un mejor desempeño en las actividades enfermeras. 
Por lo que el panorama reflejado en el presente estudio establece que el personal de enfermería que asistió a un curso taller de terapia intravenosa incremento de manera significativa el conocimiento y la práctica, con el cual se puede evidenciar la calidad de la atención, la eficiencia y la prevención de complicaciones como indicador de la profesionalización de enfermería, al implementar estrategias para el mejoramiento de procedimientos.

\section{BIBLIOGRAFÍA}

1. Manual de terapia intravenosa. 2008. Disponible en línea: http://www.osakidetza.euskadi.net/v19hgal0004/es/contenidos/informacion/hgal guias manu ales/es hgal/adjuntos/manual de terapia intravenosa.pdf (Recuperado el 24 de Septiembre de 2011).

2. Nieto, M. Accesos venosos de emergencia. En: Guía para el manejo de Urgencias. 2008. Disponible en

línea: http://www.aibarra.org/Apuntes/criticos/Guias/Enfermeria/Accesos venosos de emergencia. pdf (Recuperado el 25 de Septiembre de 2011).

3. Shah, PS; Sinha, AK. Heparina para controlar uso del catéter intravenoso periférico en neonatología. Cochranz Library Plus.Oxford.2002.

4. Norma Oficial Mexicana de Emergencia para la vigilancia epidemiológica, prevención y control de las infecciones nosocomiales. 2003. Disponible en línea: http://www.bidihmujer.salud.gob.mx/documentos/leyes/NOM-EM-002-SSA22003\%20infecciones\%20nosocomiales.pdf (Recuperado el 25 de Septiembre de 2011).

5. Leyva Ruíz, G. La enseñanza de terapia intravenosa desde una perspectiva de enseñanza situada. Memorias de Congreso. Primer Congreso Latinoamericano de Ciencias de la Educación. México: Universidad Autónoma de Baja California; 2010.

6. Secretaria de Salud (2001). Programa Nacional de Salud 2001-2006. México: Autor.

7. Secretaría de Salud (2007). Programa Nacional de Salud 2007-2012. México: Autor.

8. Polit, D; Hungler, B. Investigación científica en ciencias de la salud. México: McGraw-Hill Interamericana; 2000.

9. Resendiz Gutiérrez, MG. Conocimiento y uso de precauciones universales en la instalación de venoclisis. Rev. Desarrollo Científico de Enfermería 2004; 14 (4): 117-121.

10.Peña, VR; Rodríguez, MJ; López, JM; Martínez, MT. Naranjo, DO. Conocimiento y conductas del personal de salud sobre el lavado de manos en un servicio de emergencia. Rev. Mex. Med. Urg. 2002; 1(2): 41-43.

11. Rosales Barrera, S; Reyes Gómez, E. Fundamentos de enfermería. México: Manual Moderno; 2004.

12. Precauciones durante la atención del paciente. Disponible en línea: http://www.salud.gob.mx/conasida/guias/a med/guia14.htm. (Recuperado el 31 de Agosto de 2004)

13. García, AE; Cesar, PV. Medidas Bioseguridad, precaución estándar y sistema de aislamiento. Rev. Enfer IMSS 2002; 10 (1): 27-30.

14. Resendiz Gutiérrez, MG. Manual de terapia Intravenosa, utilizado en el curso-taller de terapia intravenosa en el Hospital General ISSSTE.

15. Rodrigo, O; Muñoz, MJ; García, S García, J. Complicaciones de los catéteres venosos periféricos en los niños según su localización. Rev. Metas de Enfermería 2003; 6 (52): 24.

16. Carvajal, J. Tesis sustentada para obtener título profesional de Licenciado en Enfermería. 2002. Disponible en línea: http://www.monografias.com/trabajos/infeccionescateter/infecciones-cateter.shtml. (Recuperado en Mayo de 2006).

17. Aguilar Eloy, M; García López, B; Hernández Tezoquipa, I; Rosas Reséndiz, MT. Las infecciones nosocomiales: registrar para prevenir. Rev Enferm IMSS 2004; 12 (2): 89-92. 
18. Organización Mundial de la Salud. Prevención de las infecciones nosocomiales. Guía práctica. Malta: Organización Mundial de la Salud; 2003.

19. Campos Pérez, FJ; Tapia Jurado, J. Punción intravenosa (inyección intravenosa, toma de muestra venosa, catéter corto y venoclisis, catéter largo y presión venosa central). México: Editorial Alfil; 2005. 